\title{
Comparación del efecto anestésico del aceite de clavo, solución salina y solución coloidal en juveniles de Chirostoma jordani (Woolman, 1894)
}

\author{
Comparison of anaesthetic effect of clove oil, saline solution and colloidal solution \\ in juvenile Chirostoma jordani (Woolman, 1894) \\ G Vázquez ${ }^{a^{*}}$, T Castro ${ }^{b}$, A Hernández ${ }^{b}$, J Castro ${ }^{b}$, R De Lara ${ }^{b}$ \\ aDoctorado en Ciencias Biológicas y de la Salud, Universidad Autónoma Metropolitana. \\ bDepartamento El Hombre y su Ambiente, Laboratorio de Producción de Alimento Vivo, \\ Universidad Autónoma Metropolitana Unidad Xochimilco. México, D.F.
}

\begin{abstract}
SUMMARY
The fish Chirostoma jordani undergoes intense stress during handling practices, making it susceptible to disease and high mortality rates. The objective of this study was to compare the anaesthetic effects of clove oil, saline solution and colloidal solution (Pentabiocare) on juvenile Chirostoma jordani. 18 C. jordani specimens aged two months old were used for each treatment. Treatments consisted on clove oil at four concentrations $(5,8,13$ and $25 \mu \mathrm{L} / \mathrm{L}$ ), saline solution at $7 \mathrm{~g} / \mathrm{L}$ and colloidal solution at $5 \mathrm{~mL} / \mathrm{L}$. The anaesthesia stages and recovery time of fish were recorded during a six hour period. High concentrations of clove oil and prolonged exposure to it were associated with deep stages of anaesthesia. Clove oil in a concentration of 25 $\mu \mathrm{L} / \mathrm{L}$ induced fish to stage three and after three hours of being exposed they entered stage five, while those sedated with saline and colloidal solutions were induced to stage one and showed the longest sedation times (six hours). Recovery time in juveniles when using $25 \mu \mathrm{L} / \mathrm{L}$ clove oil concentration was extended to 3,006.0 \pm 5.3 seconds, while fish exposed to saline and colloidal solution showed a recovery of opercular rhythm, responsiveness to external stimuli and active swimming within 60 to 180 seconds. The results indicated that colloidal and saline solution kept the $C$. jordani juveniles in a state of light sedation during a six hours period with brief intervals of recovery. The use of clove oil at 5 and $13 \mu \mathrm{L} / \mathrm{L}$ doses induced juveniles to light and deep anaesthesia stages, respectively, with a recovery time under 420 seconds.
\end{abstract}

Palabras clave: Chirostoma jordani, anestesia, aceite de clavo, solución salina.

Key words: Chirostoma jordani, anesthesia, clove oil, saline solutions.

\section{INTRODUCCIÓN}

Actualmente en la acuicultura intensiva los peces se encuentran sometidos a un mayor hacinamiento y procedimientos de manipulación durante la clasificación, captura, transporte y comercialización en vivo (Pawar y col 2011). Este manejo implica la introducción de objetos en el agua que inducen a un estrés severo, modificando la fisiología, comportamiento y bienestar de los animales, reflejados principalmente en lesiones físicas, crecimiento lento, inmunosupresión e incluso en altas mortalidades (Barton 1997, Summerfelt y Smith 1990, Kazun y Siwicki 2001, Conte 2004). Para contrarrestar el estrés se ha empleado la anestesia como una valiosa herramienta durante manejo de los peces (Coyle y col 2004).

La anestesia puede definirse como la acción de las sustancias para reducir temporalmente la sensibilidad y reflejos de los individuos. Los anestésicos ideales son aquellos que actúan rápidamente, con poca hiperactividad, a dosis bajas y con períodos cortos de recuperación

Aceptado: 25.10.2012.

* Calz. del Hueso 1100, Col. Villa Quietud. México D. F. 04960, México;gavaz@correo.xoc.uam.mx
(Ross y Ross 1999, Coyle y col 2004). En la acuicultura es indispensable elegir anestésicos con alta eficiencia, disponibilidad y bajo costo, además que no sean un factor tóxico para los peces y el personal que los aplica. La aplicación y dosis óptima del anestésico se encuentra relacionada con el comportamiento del pez, la especie, tamaño, densidad así como también de la calidad del agua (Mylonas y col 2005).

Entre los anestésicos más empleados en peces se encuentran la benzocaína, metomidato, etomidato, fenoxietanol, quinaldina (Coyle 2004), AQUI-S (isoeugenol) (Bosworth y col 2007) MS-222 (metanosulfonato de tricaína) y aceite de clavo (eugenol) (Peake 1998, García-Gómez y col 2002, Ghazilou y col 2010). La mayoría de estos compuestos no se encuentran certificados para su uso en animales de consumo humano, de acuerdo con la Food and Drug Administration (FDA) y el MS-222 es el único producto aprobado como anestesia para peces, sin embargo presenta respuestas negativas en el sistema inmunitario de los peces y su costo es elevado (Palić y col 2006).

Una opción es el uso de sustancias de origen natural, como el aceite de clavo de olor (Eugenia aromatica), cuyo compuesto activo es el eugenol (4-alil-metoxifenol), el cual es categorizado por la FDA como seguro, además de mostrar resultados satisfactorios por reducir el estrés 
e inducir a diferentes niveles de anestesia a numerosas especies acuáticas como peces, crustáceos y moluscos sin efectos negativos y con bajo costo para los acuicultores. Respecto a las dosis aplicadas de esta sustancia, varios investigadores han reportado concentraciones experimentales que van de 2 a $120 \mathrm{mg} / \mathrm{L}$ en especies como el salmón, trucha, bagre, tilapia, lobina y carpa (García-Gómez y col 2002, Cooke y col 2004, Coyle y col 2004, Guénette y col 2007). Sin embargo, en la acuicultura pocos estudios han examinado el uso de bajas concentraciones de aceite de clavo para lograr la anestesia de peces en estadios con tallas pequeñas (Perdikaris y col 2010).

Otra alternativa para reducir el estrés en peces es la sal común $(\mathrm{NaCl})$ que en los cultivos se emplea como un aditivo para mitigar el nerviosismo generado durante la manipulación y transporte, así como el estrés asociado a concentraciones altas de nitritos, debido a que las sales reducen los desequilibrios iónicos y osmorregulatorios; las concentraciones reportadas van desde 0,1 al $1 \%$. Sin embargo, la eficacia de la sal para aminorar los efectos del estrés puede variar entre especies y la gravedad de la situación estresante (Conte 2004) y su resultado como anestésico no ha sido documentado. Tal es el caso de especies de interés comercial como el bagre de canal, trucha y pejerrey (aterínido) en las que se han observado resultados favorables en la sobrevivencia, reduciendo el estrés por transporte con una rápida recuperación (Bath y Eddy 1979, Tsuzuki y col 2001).

Durante la aclimatación de especies acuáticas, otro aditivo común para controlar el estrés es la solución coloidal (Pentabiocare), donde uno de sus principales componentes es la vitamina $\mathrm{B}_{1}$ (tiamina), que funciona sobre el sistema nervioso del organismo, por lo cual está indicada para el manejo de peces (Robles-Mendoza y col 2009). Esta solución se ha empleado durante la adaptación en cautiverio de Poecilia reticulata y Skiffia bilineata (Valero y col 2009) y en el cultivo del anfibio Ambystoma mexicanum (RoblesMendoza y col 2009); sin embargo, la información sobre la dosis y su efecto como anestésico no se ha reportado.

Los peces del género Chirostoma (Atherinopsidae), conocidos en México como "pescados blancos o charales", representan un recurso sociocultural ya que forman parte de las pesquerías artesanales de los lagos en la región central del país (Martínez y col 2006), tal es el caso de las poblaciones del "charal" Chirostoma jordani, las cuales se encuentran en riesgo por la modificación de su hábitat y la sobreexplotación (Olvera-Blanco y col 2009). Una de las limitaciones en el cultivo de $C$. jordani se enfoca en la elevada susceptibilidad al estrés durante la captura, manejo y transportación ocasionando una alta tasa de mortalidad, incluso en etapas tempranas (Martínez-Palacios y col 2002). Por tanto, es de vital importancia el empleo de sustancias que reduzcan el estrés en esta especie durante su manejo, para así facilitar su cultivo y disminuir las pérdidas económicas. Existen investigaciones en la especie $C$. estor donde se han aplicado anestésicos como la benzocaína, aceite de clavo, MS 222 y 2- fenoxietanol con respuestas positivas a la anestesia entre ellos (Morales-Ventura y col 2005, Ross y col 2007), no obstante algunos de éstos son de difícil acceso y costo elevado. La respuesta de $C$. jordani a sustancias como el aceite de clavo, sal y soluciones coloidales para inducirlo a la anestesia no se ha reportado y se desconoce su respuesta en la recuperación, por tanto el objetivo de este estudio fue evaluar el efecto anestésico del aceite de clavo, solución salina y solución coloidal (Pentabiocare), durante la inducción y recuperación de juveniles de $C$. jordani en condiciones experimentales.

\section{MATERIAL Y MÉTODOS}

El experimento fue conducido en el Laboratorio de Producción de Alimento Vivo de la Universidad Autónoma Metropolitana Unidad Xochimilco, Ciudad de México, bajo la autorización y supervisión del Comité Académico de Departamento de Producción Agrícola y Animal.

\section{ORGANISMOS EXPERIMENTALES}

Se utilizaron 108 juveniles de Chirostoma jordani de dos meses de edad con las siguientes características: peso 0,18 $\mathrm{g} \pm 0,01$; longitud total $2,5 \mathrm{~cm} \pm 0,4$; altura $0,1 \mathrm{~cm} \pm 0,01$; ancho $0,4 \mathrm{~cm} \pm 0,01$ (promedio $\pm \mathrm{DE}$ ) obtenidos a partir de los reproductores del Laboratorio de Producción de Alimento Vivo, UAM Unidad Xochimilco. Previo al experimento, los peces se alimentaron con una dieta comercial para trucha ( $45 \%$ de proteína cruda, Pedregal, México) y alimento vivo (rotíferos, cladóceros y nauplios de Artemia sp), la cual fue suspendida $8 \mathrm{~h}$ antes de iniciar el ensayo.

\section{DISEÑO EXPERIMENTAL}

Los tratamientos empleados fueron aceite de clavo, solución salina y solución coloidal, los cuales se emplearon como sustancias inductoras a la anestesia. Las dosis (niveles) empleadas del aceite de clavo se consideraron de acuerdo a las concentraciones utilizadas por Ucar y Atamanalp (2010) quienes aplicaron 2,78 $\mu \mathrm{g}$ de aceite de clavo/g de peso vivo en juveniles de trucha; esta dosis se tomó como referencia para emplearla en $C$. jordani, asignando las siguientes dosis: 5, 8, 13 y $25 \mu \mathrm{L} / \mathrm{L}$. En el caso de la solución salina $(\mathrm{NaCl})$ la dosis fue de $7 \mathrm{~g} / \mathrm{L}$, considerando lo reportado por Hernández-Rubio (2006), quienes recomiendan de 5 a $10 \mathrm{~g} / \mathrm{L}$ de salinidad para el manejo de Chirostoma. La solución coloidal (Pentabiocare Biomaa, México; tiamina, ácido poliamino carbónico, coloidales protectores y tiosulfato de sodio) se aplicó en dosis de $5 \mathrm{~mL} / \mathrm{L}$, lo cual representa 2,5 veces más a lo sugerido por Valero y col (2009) para la aclimatación de guppys adultos. Las condiciones promedio del agua durante el ensayo fueron $24 \pm 2{ }^{\circ} \mathrm{C}$ de temperatura, pH $8 \pm 0,5$ y $8,5 \pm 0,3 \mathrm{mg} / \mathrm{L}$ oxígeno disuelto; los peces se 
mantuvieron con fotoperíodo de 12:12 horas luz: oscuridad y aireación constantes.

Como unidad experimental se consideró a cada pez, el cual fue alojado en un recipiente plástico con capacidad de $2 \mathrm{~L}$ con $1 \mathrm{~L}$ de agua ionizada, cada dosis de los tratamientos se realizó por triplicado, para tener así un total de 18 peces por período de exposición en los tratamientos, los cuales se distribuyeron aleatoriamente. Este procedimiento se realizó en las distintas horas de exposición a las sustancias para valorar la relación dosis respuesta al tratamiento a las 1, 2, 3, 4, 5, 6 h, después de su aplicación. El criterio de los períodos de exposición se definió considerando los tiempos empleados para realizar el manejo (captura, medición, aclimatación, transportación y distribución) de esta especie.

Para valorar el comportamiento de los peces durante la inducción y recuperación a la anestesia se emplearon los criterios descritos por Cooke y col (2004), modificados para la talla y las características de los juveniles de $C$. jordani (cuadro 1). Dentro de dichos criterios de evaluación el ritmo opercular promedio de los peces se determinó en ensayos previos obteniendo $93 \pm 8$ latidos operculares por minuto, este dato se referenció para asignar el ritmo opercular normal en los peces. Después de realizar las observaciones los peces fueron transferidos a recipientes con agua sin tratamiento para evaluar su recuperación en tiempo (segundos) con un cronómetro digital. La recuperación correspondió al período de tiempo transcurrido desde la aplicación de los tratamientos hasta que los peces reaccionaron a los siguientes criterios: 1) recuperación del ritmo opercular, 2) respuesta a estímulos externos y 3) natación activa. Las evaluaciones fueron realizadas por tres personas previamente capacitadas, quienes asignaron la fase del estado anestésico y el tiempo de recuperación en los tratamientos.

\section{ANÁLISIS ESTADÍSTICOS}

Los resultados fueron analizados con el paquete estadístico Systat 13 (Systat Software Inc.), la normalidad de las variables medidas fue comprobada mediante la prueba de Shapiro-Wilk $(\mathrm{P}<0,10)$, mientras que la homocedasticidad se confirmó empleando la prueba de Bartlett (Montgomery 1997). Dado que los datos no cumplieron con los supuestos de normalidad y homocedasticidad, los resultados se analizaron con la prueba no paramétrica de Kruskal-Wallis $(\alpha=0,05)$ y a posteriori una comparación de medias con la Prueba de Nemenyi (Zar 1996).

\section{RESULTADOS}

\section{FASE DE INDUCCIÓN}

Los tratamientos aceite de clavo, la solución salina y solución coloidal (Pentabiocare) mostraron diferencias en la fase de anestesia alcanzada por los juveniles de $C$. jordani (cuadro 2). Los peces expuestos durante la hora 1 , a los cuales se les aplicó $13 \mu \mathrm{L} / \mathrm{L}$ de aceite de clavo alcanzaron la sedación profunda (Fase 2); al usar 25 $\mu \mathrm{L} / \mathrm{L}$ éstos perdieron parcialmente el equilibrio (Fase 3 ), observando natación errática, incremento en el ritmo opercular y reactividad sólo a estímulos manuales fuertes. Sin embargo, en la segunda hora de exposición a los tratamientos, al emplear las concentraciones de 8 y $13 \mu \mathrm{L} / \mathrm{L}$ de aceite de clavo, los peces alcanzaron la fase 3 (pérdida parcial del equilibrio), y los juveniles expuestos a $25 \mu \mathrm{L} / \mathrm{L}$ perdieron totalmente el equilibrio (Fase 4); mientras que los organismos expuestos en la solución salina, solución coloidal y $5 \mu \mathrm{L} / \mathrm{L}$ de aceite de clavo mostraron efectos mínimos de anestesia (sedación ligera, Fase 1). Durante la tercer hora de contacto de los peces a $5 \mu \mathrm{L} / \mathrm{L}$ de aceite de clavo se modificó el estado de anestesia, presentándose

Cuadro 1. Criterios para la evaluación de los estados de inducción a la anestesia.

Criteria for assessing the stages of induction anaesthesia.

\begin{tabular}{|c|c|c|}
\hline Fase & Estado de anestesia & Descripción \\
\hline 0 & Normal & Reactivo a estímulos externos, ritmo opercular normal. \\
\hline 1 & Sedación ligera & $\begin{array}{l}\text { Ligera pérdida de la respuesta a estímulos, ligera disminución del ritmo opercular, } \\
\text { equilibrio normal. }\end{array}$ \\
\hline 2 & Sedación profunda & $\begin{array}{l}\text { Pérdida total de la respuesta a estímulos externos, ligera disminución del ritmo opercular, } \\
\text { equilibrio normal. }\end{array}$ \\
\hline 3 & Pérdida parcial del equilibrio & $\begin{array}{l}\text { Pérdida del equilibrio, nado errático, incremento del ritmo opercular, respuesta sólo a } \\
\text { fuertes estímulos táctiles o vibracionales. }\end{array}$ \\
\hline 4 & Pérdida total del equilibrio & Ritmo opercular lento, pérdida total del equilibrio. \\
\hline 5 & Pérdida de reflejos & Pérdida total de la respuesta estímulos, ritmo opercular lento, pérdida de todos los reflejos. \\
\hline 6 & Colapso medular & Suspensión de movimientos operculares. \\
\hline
\end{tabular}

Criterios descritos en Cooke y col (2004), adaptados para juveniles de Chirostoma jordani. 
la fase 3 (pérdida parcial del equilibrio), de igual manera se observó en la dosis de $25 \mu \mathrm{L} / \mathrm{L}$ un aumento de la respuesta a la fase 5 (pérdida de reflejos). A la cuarta hora de exposición de $5 \mu \mathrm{L} / \mathrm{L}$ de aceite de clavo la respuesta de los peces nuevamente se modificó a la fase 1 de anestesia (sedación ligera), en las dosis 8 y $13 \mu \mathrm{L} / \mathrm{L}$ los juveniles alcanzaron la fase 4 . En la quinta hora de exposición a los tratamientos los peces se mantuvieron en los mismos estados de anestesia de la hora anterior. Únicamente en la sexta hora se registró un incremento de la anestesia de C. jordani con la dosis $13 \mu \mathrm{L} / \mathrm{L}$ de aceite de clavo (Fase 5; pérdida de reflejos). La respuesta de los peces fue similar (sedación ligera) durante las seis horas de exposición al aplicar los tratamientos de solución salina y solución coloidal (cuadro 2).

\section{TIEMPO DE RECUPERACIÓN}

El tiempo de recuperación del ritmo opercular de los peces expuestos a los tratamientos mostró diferencias significativas $(\mathrm{P}<0,001)$ a partir de la segunda hora. Los juveniles en contacto con $5 \mu \mathrm{L} / \mathrm{L}$ de aceite de clavo, solución salina y solución coloidal mostraron períodos cortos de recuperación (60,3 $\pm 1,2$ a 124,3 $\pm 3,8$ segundos) durante los seis períodos de exposición, mientras que la recuperación fue mayor $(181,3 \pm 1,5$ a 306,3 \pm 3,8 segundos $)$ en los peces expuestos al resto de las concentraciones de aceite de clavo (cuadro 3). La recuperación de la respuesta a estímulos externos en $C$. jordani fue diferente $(\mathrm{P}<0,001)$ durante las horas de exposición, ya que en los tratamientos de solución salina, solución coloidal y $5 \mu \mathrm{L} / \mathrm{L}$ de aceite de clavo los juveniles se recuperaron en menor tiempo $(60,3 \pm 2,5$ a $180,3 \pm 1,5$ segundos $)$, en contraste con la adición de $25 \mu \mathrm{L} / \mathrm{L}$ de aceite de clavo donde la recuperación fue de hasta 904, $7 \pm 4,2$ segundos, a medida que se prolongó la exposición al tratamiento el tiempo de recuperación se incrementó (cuadro 3). Con respecto al tiempo de recuperación de la natación activa en los organismos, las soluciones salina y coloidal durante las seis horas de exposición presentaron un corto restablecimiento en el nado $(62,0 \pm 2,0$ a $124,3 \pm 3,8$ segundos $)$, mientras que al aplicar $25 \mu \mathrm{L} / \mathrm{L}$ de aceite de clavo los peces mostraron una recuperación más lenta (3.006,0 \pm 5,3 segundos), manifestando que a menor concentración del tratamiento con períodos cortos de exposición, la recuperación es más eficiente (cuadro 3).

\section{DISCUSIÓN}

El estrés tiene un impacto negativo en los peces en cautiverio y se encuentra intrínsecamente asociado a la acuicultura, ya sea por los procedimientos de manipulación de los peces o por la necesidad del apartamiento de éstos en el agua, factores que pueden modificar su crecimiento, agravar los estados de enfermedad o aumentar la mortalidad dificultando así el cultivo. Recientemente, diversas técnicas se han implementado para mejorar las condiciones de manejo en los cultivos de peces, tal es el caso de los anestésicos que se utilizan para reducir el estrés ocasionado por manipulación (Palić y col 2006).

En este experimento, el empleo de una concentración de $5 \mu \mathrm{L} / \mathrm{L}$ de aceite de clavo en juveniles de Chirostoma jordani los indujo a una sedación ligera durante las dos primeras horas, alcanzando en la hora tres la pérdida parcial del equilibrio, y regresando a la sedación ligera en las siguientes horas; este comportamiento fue similar a lo encontrado por Morales-Ventura y col (2005) quienes emplearon, durante el manejo, aceite de clavo para anestesiar a juveniles de Chirostoma estor y al aumentar la concentración del anestésico el tiempo de inducción al estado de anestesia se redujo, pero no para la recuperación. La sedación ligera que se observó en juveniles de $C$. jordani al emplear $5 \mu \mathrm{L} / \mathrm{L}$ de aceite de clavo se considera favorable para la acuicultura tal como lo reportan Ross y Ross (1999) en la captura y transportación de peces, donde se requiere que los organismos se mantengan sedados por lapsos largos de tiempo y éstos no sufran daño por la pérdida del equilibro. En cuanto a las dosis de aceite de clavo 8,13 y $25 \mu \mathrm{L} / \mathrm{L}, C$. jordani alcanzó en las primeras tres horas la fase tres de anestesia, presentando nado errático,

Cuadro 2. Fases de inducción a la anestesia en juveniles de Chirostoma jordani expuestos a los tratamientos con aceite de clavo, solución salina y solución coloidal.

Induction phase of anaesthesia in juvenile Chirostoma jordani exposed to treatments clove oil, saline solution and colloidal solution.

\begin{tabular}{|c|c|c|c|c|c|c|}
\hline \multirow{2}{*}{$\begin{array}{l}\text { Tiempo de exposición } \\
\text { (h) }\end{array}$} & \multicolumn{4}{|c|}{ Aceite de clavo $(\mu \mathrm{L} / \mathrm{L})$} & \multirow{2}{*}{$\begin{array}{l}\text { Solución salina } \\
7 \mathrm{~g} / \mathrm{L}\end{array}$} & \multirow{2}{*}{$\begin{array}{l}\text { Solución coloidal } \\
5 \mathrm{~mL} / \mathrm{L}\end{array}$} \\
\hline & 5 & 8 & 13 & 25 & & \\
\hline 1 & 1 & 1 & 2 & 3 & 1 & 1 \\
\hline 2 & 1 & 3 & 3 & 4 & 1 & 1 \\
\hline 3 & 3 & 3 & 3 & 5 & 1 & 1 \\
\hline 4 & 1 & 4 & 4 & 5 & 1 & 1 \\
\hline 5 & 1 & 4 & 4 & 5 & 1 & 1 \\
\hline 6 & 1 & 4 & 5 & 5 & 1 & 1 \\
\hline
\end{tabular}


Cuadro 3. Tiempo de recuperación (segundos) promedio $( \pm \mathrm{DE})$ de juveniles de Chirostoma jordani expuestos a los tratamientos con aceite de clavo y dos soluciones (salina y coloidal).

Average $( \pm \mathrm{SD})$ recovery time (seconds) in juvenile Chirostoma jordani exposed to treatments clove oil and two solutions (saline and colloidal)

\begin{tabular}{|c|c|c|c|c|c|c|}
\hline \multirow{2}{*}{$\begin{array}{l}\text { Tiempo de exposición } \\
\text { (h) }\end{array}$} & \multicolumn{4}{|c|}{ Aceite de clavo $(\mu \mathrm{L} / \mathrm{L})$} & \multicolumn{2}{|c|}{ Solución } \\
\hline & 5 & 8 & 13 & 25 & $\begin{array}{l}\text { Salina } \\
7 \mathrm{~g} / \mathrm{L}\end{array}$ & $\begin{array}{l}\text { Coloidal } \\
5 \mathrm{~mL} / \mathrm{L}\end{array}$ \\
\hline \multicolumn{7}{|c|}{ Recuperación ritmo opercular } \\
\hline 1 & $60,7 \pm 1,5^{\mathrm{a}}$ & $61,3 \pm 1,1^{\mathrm{a}}$ & $61,5 \pm 1,5^{\mathrm{a}}$ & $61,2 \pm 0,2^{\mathrm{a}}$ & $60,4 \pm 1,5^{\mathrm{a}}$ & $61,1 \pm 1,3^{\mathrm{a}}$ \\
\hline 2 & $60,3 \pm 1,2^{\mathrm{d}}$ & $182,3 \pm 3,2^{b}$ & $243,7 \pm 3,2^{\mathrm{a}}$ & $62,3 \pm 2,1^{\mathrm{d}}$ & $59,7 \pm 1,5^{\mathrm{d}}$ & $123,7 \pm 3,2^{c}$ \\
\hline 3 & $61,7 \pm 2,3^{\mathrm{d}}$ & $244,0 \pm 4,0^{\mathrm{b}}$ & $241,7 \pm 2,1^{b}$ & $306,3 \pm 3,8^{a}$ & $61,0 \pm 1,7^{\mathrm{d}}$ & $124,3 \pm 3,8^{c}$ \\
\hline 4 & $61,7 \pm 1,5^{\mathrm{c}}$ & $181,3 \pm 1,5^{\mathrm{b}}$ & $181,7 \pm 1,5^{\mathrm{b}}$ & $242,3 \pm 2,1^{a}$ & $61,7 \pm 1,5^{\mathrm{c}}$ & $63,0 \pm 2,6^{c}$ \\
\hline 5 & $62,3 \pm 2,1^{\mathrm{c}}$ & $184,0 \pm 3,6^{\mathrm{b}}$ & $185,0 \pm 4,4^{b}$ & $240,3 \pm 0,6^{\mathrm{a}}$ & $62,3 \pm 2,1^{\mathrm{c}}$ & $61,7 \pm 1,5^{\mathrm{c}}$ \\
\hline 6 & $63,3 \pm 2,5^{b}$ & $184,0 \pm 4,5^{\mathrm{a}}$ & $182,0 \pm 2,0^{\mathrm{a}}$ & $184,0 \pm 2,6^{\mathrm{a}}$ & $61,7 \pm 1,5^{b}$ & $63,7 \pm 3,2^{b}$ \\
\hline \multicolumn{7}{|c|}{ Respuesta a estímulos externos } \\
\hline 1 & $180,3 \pm 1,5^{\mathrm{a}}$ & $63,7 \pm 3,2^{\mathrm{c}}$ & $61,3 \pm 1,2^{\mathrm{c}}$ & $122,0 \pm 2,0^{b}$ & $62,3 \pm 2,1^{c}$ & $122,7 \pm 2,5^{b}$ \\
\hline 2 & $60,3 \pm 2,5^{\mathrm{d}}$ & $183,0 \pm 2,6^{\mathrm{b}}$ & $243,0 \pm 2,6^{\mathrm{a}}$ & $62,3 \pm 2,5^{\mathrm{d}}$ & $61,7 \pm 1,5^{\mathrm{d}}$ & $124,7 \pm 4,2^{c}$ \\
\hline 3 & $62,0 \pm 2,6^{e}$ & $243,3 \pm 3,1^{\mathrm{c}}$ & $362,3 \pm 2,1^{b}$ & $482,0 \pm 2,0^{\mathrm{a}}$ & $62,3 \pm 2,5^{\mathrm{e}}$ & $122,7 \pm 3,1^{\mathrm{d}}$ \\
\hline 4 & $61,0 \pm 1,0^{\mathrm{d}}$ & $182.3 \pm 2,1^{b}$ & $182,7 \pm 2,3^{b}$ & $482,7 \pm 2,3^{\mathrm{a}}$ & $62,3 \pm 2,1^{\mathrm{d}}$ & $120,3 \pm 0,6^{c}$ \\
\hline 5 & $63,3 \pm 3,1^{\mathrm{c}}$ & $181,0 \pm 1,7^{b}$ & $182,3 \pm 2,5^{b}$ & $904,7 \pm 4,2^{\mathrm{a}}$ & $63,7 \pm 3,2^{c}$ & $62,0 \pm 2,0^{c}$ \\
\hline 6 & $122,0 \pm 2,6^{c}$ & $182,3 \pm 3,2^{b}$ & $180,7 \pm 0,6^{b}$ & $601,7 \pm 1,5^{\mathrm{a}}$ & $62,7 \pm 3,1^{\mathrm{d}}$ & $62,3 \pm 2,1^{\mathrm{d}}$ \\
\hline \multicolumn{7}{|l|}{ Natación activa } \\
\hline 1 & $182,0 \pm 2,0^{\mathrm{a}}$ & $63,3 \pm 2,9^{c}$ & $122,0 \pm 2,5^{b}$ & $122,3 \pm 2,1^{b}$ & $124,3 \pm 3,8^{b}$ & $123,0 \pm 3,0^{b}$ \\
\hline 2 & $64,0 \pm 3,6^{\mathrm{d}}$ & $182,3 \pm 2,1^{b}$ & $242,3 \pm 2,1^{a}$ & $123,0 \pm 2,6^{c}$ & $62,3 \pm 2,5^{\mathrm{d}}$ & $122,0 \pm 2,6^{c}$ \\
\hline 3 & $243,3 \pm 3,1^{c}$ & $243,0 \pm 2,6^{c}$ & $363,7 \pm 3,2^{b}$ & $481,7 \pm 1,5^{\mathrm{a}}$ & $124,7 \pm 4,2^{\mathrm{d}}$ & $123,7 \pm 3,2^{d}$ \\
\hline 4 & $124,3 \pm 3,8^{\mathrm{d}}$ & $304,7 \pm 4,2^{b}$ & $182,0 \pm 1,7^{c}$ & $3.006,0 \pm 5,3^{a}$ & $65,0 \pm 4,4^{\mathrm{e}}$ & $123,0 \pm 3,6^{\mathrm{d}}$ \\
\hline 5 & $183,0 \pm 3,6^{b}$ & $183,0 \pm 2,9^{b}$ & $182,7 \pm 2,3^{b}$ & $3.002 .7 \pm 4,5^{\mathrm{a}}$ & $62,0 \pm 2,0^{\mathrm{d}}$ & $123,7 \pm 3,2^{c}$ \\
\hline 6 & $124,0 \pm 3,5^{\mathrm{c}}$ & $242,7 \pm 2,5^{b}$ & $180,3 \pm 0,6^{b}$ & $1.803,0 \pm 2,6^{a}$ & $62,3 \pm 2,1^{\mathrm{d}}$ & $121,3 \pm 1,5^{\mathrm{c}}$ \\
\hline
\end{tabular}

abc Letras distintas dentro de fila indican diferencias $(\mathrm{P}<0,05)$.

Kruskal Wallis: Ji cuadrada $=17,0 ; 5$ grados de libertad, $\mathrm{P}=0,0045$.

lo cual no es deseable en la anestesia de peces por el incremento de lesiones físicas de acuerdo con Summerfelt y Smith (1990). Por su parte, Cooke y col (2004) señalan que a medida que se incrementa la concentración de aceite de clavo (5 a $9 \mathrm{mg} / \mathrm{L}$ ) en juveniles de lobina negra (Micropterus salmoides), la profundidad de la anestesia aumenta significativamente; esta respuesta coincide con lo encontrado en este experimento con $C$. jordani al emplear dosis de 13 y $25 \mu \mathrm{L} / \mathrm{L}$ donde los juveniles alcanzaron la fase cuatro (pérdida total del equilibrio) y cinco (pérdida de reflejos) respectivamente. De igual manera, Keene y col (1998) obtuvieron una fase profunda de anestesia y pérdida rápida del equilibrio con tiempos cortos de recuperación en juveniles de trucha arcoiris (Oncorhynchus mykiss) de seis meses de edad, al emplear 15 a $30 \mathrm{mg} / \mathrm{L}$ de aceite de clavo y al aumentar la dosis y el tiempo de exposición el aceite de clavo fue letal $(30 \mathrm{mg} / \mathrm{L})$. Velisek y col (2005) aplicaron en Cyprinus carpio (carpa común) dosis mayores a las manejadas en este experimento, reportando una inducción satisfactoria con el aceite de clavo en individuos de $15 \pm 5 \mathrm{~g}$, causando diferentes estados de anestesia en relación al tiempo de exposición; al aplicar una concentración de $30 \mathrm{mg} / \mathrm{L}$ en la cual se reportó la fase uno de anestesia en menos de $5 \mathrm{~min}$ y en un período menor a 25 min entraron en la fase cuatro con un restablecimiento menor a 15 minutos, y al aumentar las concentraciones de aceite de clavo observaron estados de anestesia más profundos con tiempos de recuperación prolongados, la aplicación de más de $70 \mathrm{mg} / \mathrm{L}$ se consideró como letal. Este comportamiento de la anestesia en los peces está relacionado a la alta captación y solubilidad de eugenol a través de las branquias así como a la especie (Iversen y col 2003, Guénette y col 2007). Por su parte, Perdikaris y col (2010) mencionan que en la inducción y recuperación de la anestesia es importante considerar la actividad respiratoria de la propia especie y la talla, debido a que en un organismo con mayor actividad respiratoria se incrementará la captación de eugenol a través de las branquias.

Por otra parte, la fase ideal de anestesia según Summerfelt y Smith (1990) se logró en la dosis de $13 \mu \mathrm{L} / \mathrm{L}$ del tratamiento de aceite de clavo por un período máximo de una hora, lo cual se podría emplear en manejos cortos de 
los peces, de igual forma sucede en otras especies como Micropterus salmoides según lo reportado por Cooke y col (2004), donde señalan que en la sedación profunda (Fase 2) los organismos no presentaron daños físicos ocasionados por el contacto de los mismos peces o del tanque durante la transportación, debido a que éstos redujeron su gasto cardíaco y se mantuvieron en equilibro, lo cual es importante considerar para el manejo de $C$. jordani.

Con respecto a la recuperación que presentaron los juveniles de $C$. jordani se observa que hay una relación entre la concentración del aceite de clavo y el tiempo de recuperación, de tal manera que en la dosis de $5 \mu \mathrm{L} / \mathrm{L}$ los organismos se restablecieron en menor tiempo, en cuanto a las variables consideradas; mientras que los peces en la dosis de $25 \mu \mathrm{L} / \mathrm{L}$ tardaron hasta 50 minutos en recobrar la natación activa. Según Ackerman y col (2005) y Coyle y col (2004) consideran que un anestésico eficiente induce al organismo rápidamente (menos de tres minutos) y permite que se recupere en un corto tiempo (cinco minutos) cuando el organismo es transferido a un medio libre del anestésico, estos aspectos se observaron en el presente estudio con las concentraciones de 5 y $8 \mu \mathrm{L} / \mathrm{L}$ de aceite de clavo. Por su parte, Cooke y col (2004), reportaron que al emplear concentraciones altas de aceite de clavo (10- $20 \mathrm{mg} / \mathrm{L})$ en peces de talla grande (Micropterus salmoides) se producen niveles profundos de anestesia con recuperaciones lentas, coincidiendo con lo encontrado en $C$. jordani en la dosis de $25 \mu \mathrm{L} / \mathrm{L}$.

En relación a la solución salina, los juveniles de $C$. jor dani se mantuvieron en una sedación ligera (Fase uno) por períodos prolongados de hasta seis horas. El efecto benéfico de la solución salina moderada se ha documentado en varias especies de peces y también ha sido propuesta como un mecanismo para la reducción del estrés en la cría de peces (Schreck y col 1997). La respuesta de C. jordani con la solución salina muestra que la salinidad del agua según Martínez-Palacios y col (2004) proporciona a los peces un medio más isotónico y equilibra el intercambio osmótico.

Por su parte, Bath y Eddy (1979) mencionan que la edad y tamaño de los peces pueden afectar la respuesta al cambio de la salinidad. Tsuzuki y col (2000) y Tsuzuki y col (2001) señalan que la adición de sal al medio contribuye a la adaptación fisiológica de los peces como: la reversión de los flujos de iones en las branquias, las tasas de producción de orina, la protección contra la disminución del pH de la sangre, el equilibrio osmótico y la reducción del cortisol y glucosa en el plasma. En investigaciones realizadas con los aterínidos Chirostoma estor, Odonthestes bonarensis y $O$. hatcheri se observó que la solución salina reduce el estrés por la manipulación y además disminuye las enfermedades y mejora el crecimiento y la sobrevivencia (Martínez-Palacios y col 2004, Tsuzuki y col 2000, Tsuzuki y col 2001).

En cuanto a la solución coloidal no existen reportes de su efecto como anestésico, sin embargo se ha empleado como un reductor de estrés en especies de peces como
P. reticulata y Skiffia bilineata (Valero y col 2009) y en anfibios como Ambystoma mexicanum, obteniendo una sobrevivencia del $90 \%$ debido a que conserva la condición fisiológica en el individuo (Robles-Mendoza 2009). Por otro lado, la solución coloidal contiene tiamina, la cual es una vitamina que presenta propiedades tranquilizantes y es considerada como un relajante natural. En el caso $C$. jordani esta solución tuvo un efecto anestésico ligero en los juveniles (Fase uno). Sin embargo, este producto sólo es apto para especies destinadas al ornato o investigación, de acuerdo con la Food and Drug Administration (FDA) ya que esta agencia no aprueba las sustancias que conforman a la solución coloidal para el consumo humano (FDA 2007), por tanto para especies destinadas al consumo humano se recomienda utilizar la solución salina o el aceite de clavo.

Respecto a la recuperación de $C$. jordani, al aplicar la solución salina y coloidal fue rápida (60 a 120 segundos) incluso en tiempos prolongados de exposición (seis horas), lo cual puede explicarse debido a que la salinidad aumenta la osmolalidad sanguínea y las concentraciones de electrolitos, impidiendo que los organismos sufran una disfunción fisiológica (Tsuzuki y col 2001). Asimismo, Conte (2004) señala que la recuperación fisiológica primaria en los peces se obtiene en un lapso de seis horas a un día y la total puede durar hasta 10 días; sin embargo, indica que el estrés puede durar hasta dos semanas sin llegar a ser letal. Los resultados de este experimento indicaron que el uso de la solución salina y solución coloidal mantuvieron a los juveniles de Chirostoma jordani en una sedación ligera (Fase 1), durante un lapso de seis horas con tiempos breves de recuperación, los cuales son recomendables para la transportación y manipulación que requiera períodos extensos. Las dosis de aceite de clavo 5 y $13 \mu \mathrm{L} / \mathrm{L}$ indujeron a los juveniles a un estado de anestesia ligera y profunda respectivamente con recuperación corta, siendo útil para la manipulación de esta especie en el marcaje, biometría, aplicación de vacunas o medicamentos.

\section{RESUMEN}

Los peces de la especie Chirostoma jordani presentan un intenso estrés durante las prácticas de manejo, dejándolos susceptibles a enfermedades y a tasas altas de mortalidad. El objetivo del presente estudio fue comparar el efecto anestésico del aceite de clavo, solución salina y una solución coloidal (Pentabiocare) en juveniles de Chirostoma jordani. Para cada tratamiento se utilizaron 18 ejemplares de $C$. jordani de dos meses de edad. Los tratamientos constaron en aceite de clavo (5, $8,13$ y $25 \mu \mathrm{L} / \mathrm{L})$, solución salina (7 g/L) y solución coloidal $(5 \mathrm{~mL} / \mathrm{L})$. Durante seis horas se registraron, en los peces, las fases de inducción a la anestesia y los tiempos de recuperación. Las concentraciones altas y los tiempos prolongados de exposición al aceite de clavo se asociaron a las fases profundas de la anestesia. La concentración de $25 \mu \mathrm{L} / \mathrm{L}$ de aceite de clavo indujo a los organismos a la fase tres y a partir de la tercer hora de exposición éstos entraron en la fase cinco, mientras que las soluciones salina y coloidal indujeron a los peces a la fase uno de sedación mostrando los lapsos más prolongados (seis horas). El tiempo de recuperación de los juveniles en la concentración de $25 \mu \mathrm{L} / \mathrm{L}$ de aceite de clavo se extendió a 3.006,0 \pm 5,3 segundos, mientras que con solución salina y coloidal, la respuesta de los peces a la recuperación del 
ritmo opercular, reactividad a estímulos externos y natación activa fue entre 60 a 180 segundos. Los resultados de este experimento indicaron que la solución salina y solución coloidal mantuvieron a los juveniles de $C$. jordani en una sedación ligera durante un lapso de seis horas con tiempos breves de recuperación. El uso de aceite de clavo en dosis de 5 y $13 \mu \mathrm{L} / \mathrm{L}$ indujo a los juveniles a estados de anestesia ligera y profunda respectivamente, con una recuperación de no más de 420 segundos.

\section{AGRADECIMIENTOS}

Este trabajo forma parte de la Tesis Doctoral del primer autor inscrita al Doctorado en Ciencias Biológicas y de la Salud. Universidad Autónoma Metropolitana Unidad Xochimilco. Financiamiento CONACYT-México y Proyecto Acuerdos del Rector General 2008 (UAM-Acuerdo 10).

\section{REFERENCIAS}

Ackerman PA, JD Morgan, Iwama GK. 2005. Anesthetics. Canadian Council on Animal Care, Ottawa, Canada.

Barton BA. 1997. Stress in finfish: past, present and future-a historical perspective. In: Iwama GK, Pickering AD, Sumpter JP, Schreck CB (eds). Fish Stress and Health in Aquaculture. Series 62. Society for Experimental Biology Seminar, Cambridge University Press, Cambridge, UK, Pp 1-33.

Bath RN, FB Eddy. 1979. Salt and water balance in rainbow trout (Salmo gairdneri) rapidly transferred from fresh water to sea water. $J$ Exp Biol 83, 193-202.

Bosworth BG, B Small, D Gregory, J Kim, S Black. 2007. Effects of rested-harvest using the anesthetic AQUI-S ${ }^{\mathrm{TM}}$ on channel catfish, Ictalurus punctatus, physiology and fillet quality. Aquaculture 262, 302-318

Conte SF. 2004. Stress and the welfare of cultured fish. Appl Anim Behav Sci 86, 205-223.

Cooke JS, DC Suski, GK Ostranda, LB Tuftsb, HD Wahl. 2004. Behavioral and physiological assessment of low concentrations of clove oil anaesthetic for handling and transporting largemouth bass (Micropterus salmoides). Aquaculture 239, 509-529.

Coyle SD, RM Durborow, JH Tidwell. 2004. Anesthetics in Aquaculture. Southern Regional Aquaculture Center No 3900, Kentucky State University Aquaculture Research Center, Texas, USA.

FDA, Food and Drug Administration. 2007. Guidance for Industry: Concerns related to the use of clove oil as an anesthetic for fish. Department of Health and Human Services Food and Drug Administration, Center for Veterinary Medicine, Rockville, USA.

García-Gómez A, F De la Gándara, T Raja. 2002. Utilización del aceite de clavo, Syzygium aromaticum L. (Merr. \& Perry), como anestésico eficaz y económico para labores rutinarias de manipulación de peces marinos cultivados. Boletín del Instituto Español Oceanografía $18,21-23$

Ghazilou A, HH Saeedi, F Chenary, A Nateghi, N Haghi, RS Mohammad. 2010. The anesthetic efficiency of clove oil in Caspian salmon, Salmo trutta caspius $\mathrm{K}$., smolts in dosage-salinity-pH linked approach. $J$ World Aquacult Soc 41, 655-660.

Guénette SA, FC Uhland, P Hélie, F Beaudry, P Vachon. 2007. Pharmacokinetics of eugenol in rainbow trout (Oncorhynchus mykiss). Aquaculture 266, 262-265.

Hernández-Rubio MC, G Figueroa-Lucero, IA Barriga-Sosa, JL Arredondo-Figueroa, T Castro-Barrera. 2006. Early development of the shortfin silverside Chirostoma humboldtianum (Valenciennes, 1835) (Atheriniformes: Atherinopsidae). Aquaculture 26, 1440-1446.

Iversen M, B Finstad, RS McKinley, R Eliassen. 2003. The efficacy of metomidate, clove oil, Aqui-S and benzoak as anesthetics in Atlantic salmon (Salmo salar L.) smolts, and their potential stress-reducing capacity. Aquaculture 221, 549-566.
Kazun K, AK. Siwicki 2001. Propiscin. A safe new anesthetic for fish. Arch Polish Fish 9, 183-190.

Keene JL, DLG Noakes, RD Moccia, CG Soto. 1998. The efficacy of clove oil as an anaesthetic for rainbow trout, Oncorhynchus mykiss (Walbaum). Aquac Res 29, 89-101.

Martínez-Palacios CA, E Barriga Tovar, JF Taylor MG, Ríos-Durán, LG Ross. 2002. Effect of temperature on growth and survival of Chirostoma estor estor, Jordan 1879, monitored using a simple video technique for remote measurement of length and mass of larval and juvenile fishes. Aquaculture 209, 369-377.

Martínez-Palacios CA, J Comas-Morte, JA Tello-Ballinas, M ToledoCuevas, LG Ross. 2004. The effects of saline environments on survival and growth of eggs and larvae of Chirostoma estor estor Jordan 1879 (Pisces: Atherinidae). Aquaculture 238, 509-522.

Martínez PCA, IS Racotta, MG Ríos-Durán, E Palacios, M ToledoCuevas, LG Ross. 2006. Advances in applied research for the culture of Mexican silversides (Chirostoma, Atherinopsidae). Biocell 30, 137-148.

Montgomery DC. 1997. Design and analysis of experiments. John Wiley \& Sons, New York, USA.

Morales-Ventura J, M Hernández-Martínez, M Ramírez-Flores. 2005. Empleo de la esencia de clavo y el 2-Fenoxietanol como anestésicos en el pescado blanco (Chirostoma estor). Memorias XVIII Congreso Nacional de Zoología, Monterrey Nuevo León, México, Pp 158.

Mylonas CC, G Cardinaletti, I Sigelakia, A Polzonetti-Magnib. 2005. Comparative efficacy of clove oil and 2-phenoxyethanol as anesthetics in the aquaculture of European sea bass (Dicentrarchus labrax) and gilthead sea bream (Sparus aurata) at different temperatures. Aquaculture 246, 467-481.

Olvera-Blanco YM, JL Gómez-Márquez, B Peña-Mendoza, MT GasparDillanes, C Pérez. 2009. Reproductive biology of Menidia jordani (Atheriniformes: Atherinopsidae) in Xochimilco Lake, Mexico. Ciencia Pesquera 17, 65-75.

Pali D, DM Herolt, CB Andreasen, BW Menzel, JA Roth. 2006. Anesthetic efficacy of tricaine methanesulfonate, metomidate and eugenol: Effects on plasma cortisol concentration and neutrophil function in fathead minnows (Pimephales promelas Rafinesque, 1820). Aquaculture 254, 675-685.

Pawar HB, SV Sanaye, RA Sreepada, V Harish, U Suryavanshi, ZA Tanu. 2011. Comparative efficacy of four anaesthetic agents in the yellow seahorse, Hippocampus kuda (Bleeker, 1852). Aquaculture $311,155-161$

Peake S. 1998. Sodium bicarbonate and clove oil as potential anesthetics for nonsalmonid fishes. N Amer J Fish Man 18, 919-924.

Perdikaris C, C Nathanailides, E Gouva, UU Gabriel, K Bitchava, F Athanasopoulou, A Paschou, I Paschos. 2010. Size-relative effectiveness of clove oil as an anaesthetic for rainbow trout (Oncorhynchus mykiss Walbaum, 1792) and goldfish (Carassius auratus Linnaeus, 1758). Acta Vet Brno 79, 481-490.

Robles-Mendoza C, E García-Basilio, RC Vanegas-Pérez. 2009. Maintenance media for the axolotl Ambystoma mexicanum juveniles (Amphibia: Caudata). Hidrobiologica 19, 205-210.

Ross LG, B Ross. 1999. Anaesthetic and sedative techniques for aquatic animals. Blackwell Science Ltd, Oxford, UK.

Ross LG, BJ Sanchez, C Martínez-Palacios, IS Racotta, CM Toledo. 2007. Anaesthesia, sedation and transportation of juvenile Menidia estor (Jordan) using benzocaine and hypothermia. Aquac Res 38, 909-917.

Schreck CB, BL Olla, MW Davis. 1997. Behavior responses to stress. In: Iwama GK, Pickering AD, Sumpter JP, Schreck CB (eds). Fish Stress and Health in Aquaculture. Society of Experimental Biology Seminary Series 62, Cambridge University Press, Cambridge, UK, Pp 497-541.

Summerfelt RC, LS Smith. 1990. Anaesthesia, surgery and related techniques. In: Schreck CB, Moyle PB (eds). Methods for Fish Biology. American Fisheries Society, Bethesda, MD, USA, Pp 213-272. 
Tsuzuki MY, H Aikawa, CA Strussman, F Takashima. 2000. Comparative survival and growth of embryos, larvae and juveniles of pejerrey Odontesthes bonariensis and $O$. hatcheri at different salinities. $J$ Appl Ichthyol 16, 126-130.

Tsuzuki, MY, K Ogawa, CA Strussman, M Maita, F Takashima. 2001. Physiological responses during stress and subsequent recovery at different salinities in adult pejerrey Odontesthes bonariensis. Aquaculture 200, 349-362.

Ucar A, M Atamanalp. 2010. The effects of natural (clove oil) and synthetical (2-phenoxyethanol) anesthesia substances on hematology parameters of rainbow trout (Oncorhynchus mykiss) and Brown Trout (Salmo trutta fario). J Anim Vet Adv 9, 1925-1933.

Valero A, AE Magurran, GC Macías. 2009. Guppy males distinguish between familiar and unfamiliar females of a distantly related species Anim Behav 78, 441-445.

Velisek J, Z Svobodova, V Piackova, L Groch, L Nepejchalova. 2005. Effects of clove oil anaesthesia on common carp (Cyprinus carpio L.). Vet Med-Czech 50, 269-275.

Zar J. 1996. Biostatistical Analysis. Prentice-Hall, Englewood Cliffs, New Jersey, USA. 